International Journal of Pure and Applied Mathematics

Volume 94 No. 2 2014, 163-174

ISSN: 1311-8080 (printed version); ISSN: 1314-3395 (on-line version)

url: http://www.ijpam.eu

doi: http://dx.doi.org/10.12732/ijpam.v94i2.4

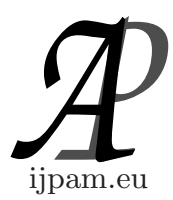

\title{
OSCILLATION CRITERIA FOR SECOND-ORDER NONLINEAR IMPULSIVE DIFFERENTIAL EQUATIONS WITH PERTURBATION
}

\author{
E. Thandapani ${ }^{1}$, K. Manju ${ }^{2}$ \\ ${ }^{1,2}$ Ramanujan Institute for Advanced Study in Mathematics \\ University of Madras \\ Chennai, 600 005, INDIA
}

\begin{abstract}
In this paper, we present some new sufficient conditions for the oscillation of all solutions of a second order perturbed differential equation with impulses. These results extend some results for the differential equation without impulses. Examples are provided to illustrate our results.
\end{abstract}

AMS Subject Classification: $34 \mathrm{~A} 37,34 \mathrm{C} 10$

Key Words: oscillation criteria, second-order differential equations, impulse effect, perturbations

\section{Introduction}

In this paper, we are concerned with the oscillatory behavior of solutions of impulsive differential equations of the form

$$
\left\{\begin{array}{l}
\left(r(t)\left(\psi(u(t))\left|u^{\prime}(t)\right|^{\alpha-1} u^{\prime}(t)\right)^{\prime}+Q(t, u(t))=P\left(t, u(t), u^{\prime}(t)\right), t \neq \theta_{k}\right. \\
\left.\Delta\left(r(t) \psi(u(t))\left|u^{\prime}(t)\right|^{\alpha-1} u^{\prime}(t)\right)\right|_{t=\theta_{k}}+Q_{k}\left(\theta_{k}, u\right)=0, t \in \mathbb{R}^{+}, k \in \mathbb{N}
\end{array}\right.
$$

where $t \geq t_{0},\left.\Delta z(t)\right|_{t=\theta}=z\left(\theta^{+}\right)-z\left(\theta^{-}\right)$and $z\left(\theta^{ \pm}\right):=\lim _{t \rightarrow \theta^{ \pm}} z(t)$. For convenience we assume $z(\theta)=z\left(\theta^{-}\right)$.

Received: February 3, 2014

(C) 2014 Academic Publications, Ltd.

${ }^{\S}$ Correspondence author url: www.acadpubl.eu 
Let $P L C(J, \mathbb{R})$ denote the set of all real valued functions $u(t)$ defined on $J \subset\left[t_{0}, \infty\right)$ such that $u(t)$ is continuous for all $t \in J$ except possibly at $t=\theta_{k}$, where $u\left(\theta_{k}^{ \pm}\right)$exist and $u\left(\theta_{k}\right)=u\left(\theta_{k}^{-}\right)$. Throughout this paper, according to the results, we assume the following conditions hold:

(H1) $r:\left[t_{0}, \infty\right) \rightarrow(0, \infty)$ is a continuously differentiable function, $\psi: \mathbb{R} \rightarrow \mathbb{R}^{+}$ is a continuously differentiable function, and $\alpha$ is a positive real number.

(H2) $Q \in P L C\left(\left[t_{0}, \infty\right) \times \mathbb{R}, \mathbb{R}\right)$, and there exists a continuous function $q$ : $\left[t_{0}, \infty\right) \rightarrow(0, \infty)$ and a continuously differentiable function $f: \mathbb{R} \rightarrow \mathbb{R}$ such that $x f(x)>0$ and $\frac{Q(t, x)}{f(x)} \geq q(t)$ for $x \neq 0$.

(H3) $P \in P L C\left(\left[t_{0}, \infty\right) \times \mathbb{R} \times \mathbb{R}, \mathbb{R}\right)$, and there exists a continuous function $p:\left[t_{0}, \infty\right) \rightarrow(0, \infty)$ such that $\frac{P(t, x, y)}{f(x)} \leq p(t)$.

(H4) $P \in P L C\left(\left[t_{0}, \infty\right) \times \mathbb{R} \times \mathbb{R}, \mathbb{R}\right)$, and there exists a continuous function $p:\left[t_{0}, \infty\right) \rightarrow(0, \infty)$ such that $\frac{P(t, x, y)}{f(x)} \leq p(t)|y|^{\alpha-1} y$ for $x \neq 0, y \neq 0$.

By a solution of equation (1) on an interval $J \subset \mathbb{R}$, we mean a continuously differentiable function $u(t)$ which is defined on $J$ such that

$$
u^{\prime}(t),\left(r(t) \psi(u)\left|u^{\prime}(t)\right|^{\alpha-1} u^{\prime}(t)\right) \in P L C(J, \mathbb{R})
$$

and which satisfies the equation (1). We consider only those solutions $u(t)$ of equation (1) which satisfy $\sup \left\{|u(t)|: t \geq T_{u}\right\}>0$ for all $T_{u} \geq t_{0}$. That is equation (1) has a solution which is nontrivial for large $t$. Such a solution of equation (1) is called oscillatory if it has arbitrarily large zeros; Otherwise, it is said to be nonoscillatory.

In recent years the oscillation theory of impulsive differential equations emerging as an important area of research, since such equations have applications in control theory, physics, biology and population dynamics. There are few papers have devoted to the oscillation criteria of second order differential equations with impulses see for example $[4,5,8]$ and the references cited therein. In $[1,6,7,10,11]$ the authors obtained some oscillation results for special cases of equation (1) without impulse effect.

Motivated by this observation, in this paper, we present sufficient conditions for the oscillation of all solutions of equation (1). The results obtained in this paper extend that of in [7] and [8]. 


\section{Main Results}

In this section we present some sufficient condition for the oscillation of all solutions of equation (1). We begin with the following theorem.

Theorem 1. Assume that $q(t)-p(t) \geq 0$ for all $t \geq t_{0}$ and conditions $\left(H_{1}\right),\left(H_{2}\right),\left(H_{3}\right)$ hold. Suppose that there exists a constant $L>0$ such that

$$
\frac{f^{\prime}(x)}{\left(\psi(x)|f(x)|^{\alpha-1}\right)^{\frac{1}{\alpha}}} \geq L \text { for } x \neq 0 .
$$

If there exists a non-increasing differentiable function $\rho:\left[t_{0}, \infty\right) \rightarrow \mathbb{R}^{+}$such that

$$
\limsup _{t \rightarrow \infty} \int_{t_{0}}^{t}\left(\frac{1}{\rho^{\alpha}(s) r(s)}\right)^{\frac{1}{\alpha}} d s=\infty
$$

and

$$
\limsup _{t \rightarrow \infty}\left[\int_{t_{0}}^{t} R(s) d s+\sum_{t_{0} \leq \theta_{k}<t} \rho^{\alpha}\left(\theta_{k}\right) q\left(\theta_{k}\right)\right]=\infty
$$

where

$$
R(t)=\rho^{\alpha}(t)\left[q(t)-p(t)-\mu r(t)\left(\frac{\rho^{\prime}(t)}{\rho(t)}\right)^{\alpha+1}\right] \text { and }
$$

$\mu:=\left(\frac{\alpha}{\alpha+1}\right)^{\alpha+1}\left(\frac{\alpha}{L}\right)^{\alpha}$, then every solution of equation (1) is oscillatory.

Proof. Suppose that there exist a non-oscillatory solution $u(t)$ of equation (1). We may assume that $u(t)$ is eventually positive. (The case $u(t)$ eventually negative is similar, so that we can omit it.) Define

$$
\omega(t)=\frac{\rho^{\alpha}(t) r(t) \psi(u(t))\left|u^{\prime}(t)\right|^{\alpha-1} u^{\prime}(t)}{f(u(t))}, t \neq \theta_{k}
$$

and

$$
\left.\Delta \omega(t)\right|_{t=\theta_{k}}=-\frac{\rho^{\alpha}\left(\theta_{k}\right) Q_{k}\left(\theta_{k}, u\left(\theta_{k}\right)\right)}{f\left(u\left(\theta_{k}\right)\right)} .
$$

Differentiating (5) and using $\left(H_{2}\right),\left(H_{3}\right)$ and equation (1), we have

$$
\begin{gathered}
\omega^{\prime}(t) \leq \alpha \frac{\rho^{\prime}(t)}{\rho(t)}|\omega(t)|-\frac{\rho^{\alpha}(t) r(t) \psi(u(t))\left|u^{\prime}(t)\right|^{\alpha-1}\left(u^{\prime}(t)\right)^{2} f^{\prime}(u(t))}{f^{2}(u(t))} \\
-\rho^{\alpha}(t)(q(t)-p(t)) .
\end{gathered}
$$


From (2) and (7), we obtain,

$$
\omega^{\prime}(t) \leq \alpha \frac{\rho^{\prime}(t)}{\rho(t)}|\omega(t)|-\rho^{\alpha}(t)(q(t)-p(t))-L \frac{|\omega(t)|^{\frac{\alpha+1}{\alpha}}}{\rho(t)(r(t))^{\frac{1}{\alpha}}}, t \neq \theta_{k} .
$$

By using the inequality

$$
A x-B x^{1+\frac{1}{\alpha}} \leq \frac{\alpha^{\alpha}}{(\alpha+1)^{\alpha+1}} A^{\alpha+1} B^{-\alpha}, A \geq 0, B>0, x \geq 0
$$

we get from (8) that

$$
\omega^{\prime}(t) \leq-\rho^{\alpha}(t)\left[q(t)-p(t)-\mu r(t)\left(\frac{\rho^{\prime}(t)}{\rho(t)}\right)^{\alpha+1}\right] .
$$

Using

$$
\int_{t_{1}}^{t} \omega^{\prime}(s) d s=\omega(t)-\omega\left(t_{1}\right)-\sum_{t_{1} \leq \theta_{k}<t} \Delta \omega\left(\theta_{k}\right),
$$

and (6), then integrating the inequality (10) from $t_{1}$ to $t$, we obtain

$$
\begin{aligned}
\omega(t) \leq \omega\left(t_{1}\right)- & \left(\int_{t_{1}}^{t} \rho^{\alpha}(s)\left[q(s)-p(s)-\mu r(s)\left(\frac{\rho^{\prime}(s)}{\rho(s)}\right)^{\alpha+1}\right] d s\right. \\
& +\sum_{t_{1} \leq \theta_{k}<t} q_{k}\left(\theta_{k}\right) \rho^{\alpha}\left(\theta_{k}\right)
\end{aligned}
$$

That is,

$$
\omega(t) \leq \omega\left(t_{1}\right)-\left[\int_{t_{1}}^{t} R(s) d s+\sum_{t_{1} \leq \theta_{k}<t} q_{k}\left(\theta_{k}\right) \rho^{\alpha}\left(\theta_{k}\right)\right] .
$$

Taking limsup in the last inequality, we obtain $\omega(t) \rightarrow-\infty$ in view of (4). Hence there exist $t_{2} \geq t_{1}$ such that $u^{\prime}(t)<0$ for $t \geq t_{2}$.

Now $q(t)-p(t) \geq 0$ and $\rho^{\alpha}(t)>0$ for all $t \geq t_{0}$. Hence

$$
\int_{t_{3}}^{t} \rho^{\alpha}(s)[q(s)-p(s)] d s \geq 0
$$

for all $t \geq t_{3}>t_{2}$. Multiplying equation (1) by $\rho^{\alpha}(t)$, we get

$$
-\rho^{\alpha}(t)\left(r(t) \psi(u(t))\left(-u^{\prime}(t)\right)^{\alpha}\right)^{\prime} \leq-\rho^{\alpha}(t)(q(t)-p(t)) f(u(t)) .
$$


Now

$$
\begin{aligned}
\left(-\rho^{\alpha}(t) r(t) \psi(u(t))\left(-u^{\prime}(t)\right)^{\alpha}\right)^{\prime}=- & \rho^{\alpha}(t)\left(r(t) \psi(u(t))\left(-u^{\prime}(t)\right)^{\alpha}\right)^{\prime} \\
& -\left(\rho^{\alpha}(t)\right)^{\prime}\left(r(t) \psi(u(t))\left(-u^{\prime}(t)\right)^{\alpha}\right) .
\end{aligned}
$$

Integrating the last inequality from $t_{3}$ to $t$ and using (13), we get

$$
\begin{aligned}
-\rho^{\alpha}(t) & r(t) \psi(u(t))\left(-u^{\prime}(t)\right)^{\alpha}-\rho^{\alpha}\left(t_{3}\right) r\left(t_{3}\right) \psi\left(u\left(t_{3}\right)\right)\left(-u^{\prime}\left(t_{3}\right)\right)^{\alpha} \\
& \leq-\int_{t_{3}}^{t}\left(\rho^{\alpha}(s)\right)^{\prime} r(s) \psi(u(s))\left(-u^{\prime}(s)\right)^{\alpha} d s \\
& -\int_{t_{3}}^{t} \rho^{\alpha}(s)(q(s)-p(s)) f(u(s)) d s-\sum_{t_{3} \leq \theta_{k}<t} \rho^{\alpha}\left(\theta_{k}\right) Q\left(\theta_{k}, u\right) .
\end{aligned}
$$

Now,

$$
\begin{aligned}
\rho^{\alpha}(t) r(t) \psi(u(t))\left(-u^{\prime}(t)\right)^{\alpha} \geq & M+\int_{t_{3}}^{t}\left(\rho^{\alpha}(s)\right)^{\prime} r(s) \psi(u(s))\left(-u^{\prime}(s)\right)^{\alpha} d s \\
& +\int_{t_{3}}^{t} \rho^{\alpha}(s)(q(s)-p(s)) f(u(s)) d s \\
& +\sum_{t_{3} \leq \theta_{k}<t} \rho^{\alpha}\left(\theta_{k}\right) q\left(\theta_{k}\right) f\left(\theta_{k}\right)
\end{aligned}
$$

where $M=\rho^{\alpha}\left(t_{3}\right) r\left(t_{3}\right) \psi\left(u\left(t_{3}\right)\right)\left(-u^{\prime}\left(t_{3}\right)\right)^{\alpha}>0$.

$$
\begin{gathered}
\rho^{\alpha}(t) r(t) \psi(u(t))\left(-u^{\prime}(t)\right)^{\alpha} \geq M+\int_{t_{3}}^{t} \rho^{\alpha}(s)(q(s)-p(s)) f(u(s)) d s \\
+\sum_{t_{3} \leq \theta_{k}<t} \rho^{\alpha}\left(\theta_{k}\right) q\left(\theta_{k}\right) f\left(\theta_{k}\right) \\
=M+f(u(t)) \int_{t_{3}}^{t} \rho^{\alpha}(s)(q(s)-p(s)) d s \\
-\int_{t_{3}}^{t} f^{\prime}(u(s)) u^{\prime}(s)\left(\int_{t_{3}}^{s} \rho^{\alpha}(v)(q(v)-p(v)) d v\right) d s \\
+\sum_{t_{3} \leq \theta_{k}<t} \rho^{\alpha}\left(\theta_{k}\right) q\left(\theta_{k}\right) f\left(\theta_{k}\right) .
\end{gathered}
$$

That is,

$$
\begin{gathered}
\rho^{\alpha}(t) r(t) \psi(u(t))\left(-u^{\prime}(t)\right)^{\alpha} \geq M, \\
\psi(u(t))\left(-u^{\prime}(t)\right)^{\alpha} \geq \frac{M}{\rho^{\alpha}(t) r(t)},
\end{gathered}
$$




$$
(\psi(u(t)))^{\frac{1}{\alpha}}\left(-u^{\prime}(t)\right) \geq\left(\frac{M}{\rho^{\alpha}(t) r(t)}\right)^{\frac{1}{\alpha}}
$$

Integrating from $t_{3}$ to $t$, by using (11), we obtain

$$
\begin{aligned}
\int_{t_{3}}^{t} \psi(u(s))^{\frac{1}{\alpha}} u^{\prime}(s) d s & \leq-(M)^{\frac{1}{\alpha}} \int_{t_{3}}^{t}\left(\frac{1}{\rho^{\alpha}(s) r(s)}\right)^{\frac{1}{\alpha}} d s \\
\int_{u\left(t_{3}\right)}^{u(t)}(\psi(y))^{\frac{1}{\alpha}} d y & \leq-(M)^{\frac{1}{\alpha}} \int_{t_{3}}^{t}\left(\frac{1}{\rho^{\alpha}(s) r(s)}\right)^{\frac{1}{\alpha}} d s
\end{aligned}
$$

Since $0<u(t) \leq u\left(t_{3}\right)$, we have

$$
\int_{u\left(t_{3}\right)}^{u\left(t_{3}\right)}(\psi(y))^{\frac{1}{\alpha}} d y \leq-(M)^{\frac{1}{\alpha}} \int_{t_{3}}^{t}\left(\frac{1}{\rho^{\alpha}(s) r(s)}\right)^{\frac{1}{\alpha}} d s
$$

or

$$
0 \leq-(M)^{\frac{1}{\alpha}} \int_{t_{3}}^{t}\left(\frac{1}{\rho^{\alpha}(s) r(s)}\right)^{\frac{1}{\alpha}} d s
$$

Now taking limit as $t \rightarrow \infty$ and using (3), we get a contradiction. The proof is complete.

From Theorem 1, we have the following corollary.

Corollary 2. Assume all conditions of Theorem 1are satisfied except (4). If

$$
\limsup _{t \rightarrow \infty}\left[\int_{t_{0}}^{t} \rho^{\alpha}(s)(q(s)-p(s)) d s+\sum_{t_{0} \leq \theta_{k}<t} \rho^{\alpha}\left(\theta_{k}\right) q\left(\theta_{k}\right)\right]=\infty
$$

and

$$
\limsup _{t \rightarrow \infty} \int_{t_{0}}^{t} \rho^{\alpha}(s) r(s)\left(\frac{\rho^{\prime}(s)}{\rho(s)}\right)^{\alpha+1} d s<\infty
$$

then every solution of equation (1) is oscillatory.

Proof. Since (14) and (15) imply (4), proof follows from Theorem 1.

Theorem 3. Assume that $q(t)-p(t) \geq 0$ for all $t \geq t_{0}$ and $\left(H_{1}\right),\left(H_{2}\right),\left(H_{3}\right)$ and (2) hold. If there exists a non-increasing differentiable function $\rho:\left[t_{0}, \infty\right) \rightarrow$ $\mathbb{R}^{+}$with (3) holds, and

$$
\liminf _{t \rightarrow \infty}\left[\int_{t_{0}}^{t} R(s) d s+\sum_{t_{0} \leq \theta_{k}<t} \rho^{\alpha}\left(\theta_{k}\right) q\left(\theta_{k}\right)\right] \geq 0
$$


where $R(t)$ as in Theorem 1, and

$$
\lim _{t \rightarrow \infty} \int_{t_{0}}^{t}\left(\frac{1}{r(s) \rho^{\alpha}(s)}\left[\int_{s}^{\infty} R(u) d u+\sum_{s \leq \theta_{k}<\infty} \rho^{\alpha}\left(\theta_{k}\right) q\left(\theta_{k}\right) \mid\right)^{\frac{1}{\alpha}} d s=\infty,\right.
$$

and

$$
\int_{\epsilon}^{\infty}\left(\frac{\psi(y)}{f(y)}\right)^{\frac{1}{\alpha}} d y<\infty, \int_{-\epsilon}^{-\infty}\left(\frac{\psi(y)}{f(y)}\right)^{\frac{1}{\alpha}} d y<\infty,
$$

then every solution of equation (1) is oscillatory.

Proof. Let $u(t)$ be a non-oscillatory solution on $\left[t_{0}, \infty\right)$ of equation (1). Without loss of generality, we may assume that $u(t)$ is eventually positive. We consider the following three cases for the behavior of $u^{\prime}(t)$.

Case 1: $u^{\prime}(t)>0$ for $t \geq t_{1}$ for some $t_{1} \geq t_{0}$. Proceeding as in the proof of Theorem 1 we get (12)

$$
\int_{t_{1}}^{t} R(s) d s+\sum_{t_{1} \leq \theta_{k}<t} \rho^{\alpha}\left(\theta_{k}\right) q\left(\theta_{k}\right) \leq \omega\left(t_{1}\right)-\omega(t) .
$$

Hence for all $t \geq t_{1}$

$$
\int_{t}^{\infty} R(s) d s+\sum_{t \leq \theta_{k}<\infty} \rho^{\alpha}\left(\theta_{k}\right) q\left(\theta_{k}\right) \leq \frac{\rho^{\alpha}(t) r(t) \psi(u(t))\left(u^{\prime}(t)\right)^{\alpha}}{f(u(t))} .
$$

Dividing the last inequality by $\rho^{\alpha}(t) r(t)$ and then integrating from $t_{1}$ to $t$, we have

$$
\begin{aligned}
\int_{t_{1}}^{t}\left(\frac{1}{r(s) \rho^{\alpha}(s)} \int_{s}^{\infty} R(u) d u\right. & +\sum_{s \leq \theta_{k}<\infty} \rho^{\alpha}\left(\theta_{k}\right) q\left(\theta_{k}\right){ }^{\frac{1}{\alpha}} d s \\
& \leq \int_{t_{1}}^{t}\left(\frac{\psi(u(s))}{f(u(s))}\right)^{\frac{1}{\alpha}} u^{\prime}(s) d s \\
& \leq \int_{u\left(t_{1}\right)}^{u(t)}\left(\frac{\psi(y)}{f(y)}\right)^{\frac{1}{\alpha}} d y .
\end{aligned}
$$

Letting $t \rightarrow \infty$, from (17) and (18), we obtain a contradiction.

Case 2: $u^{\prime}(t)$ changes sign, then there exists a sequence $\left(\alpha_{n}\right) \rightarrow \infty$ in $\left[t_{0}, \infty\right)$ such that $u^{\prime}\left(\alpha_{n}\right)<0$. Choose $n$ large enough so that

$$
\liminf _{t \rightarrow \infty}\left[\int_{\alpha_{n}}^{t} R(s) d s+\sum_{\alpha_{n} \leq \theta_{k}<t} \rho^{\alpha}\left(\theta_{k}\right) q\left(\theta_{k}\right)\right] \geq 0 .
$$


From (12), we have

$$
\omega(t) \leq \omega\left(t_{1}\right)-\left(\int_{t_{1}}^{t} R(s) d s+\sum_{t_{1} \leq \theta_{k}<t} \rho^{\alpha}\left(\theta_{k}\right) q\left(\theta_{k}\right)\right),
$$

If $t \geq \alpha_{n}$, then from

$$
\omega(t) \leq \omega\left(\alpha_{n}\right)-\left[\int_{\alpha_{n}}^{t}\left(R(s) d s+\sum_{\alpha_{N} \leq \theta_{k}<t} \rho^{\alpha}\left(\theta_{k}\right) q\left(\theta_{k}\right)\right)\right],
$$

we have

$$
\liminf _{t \rightarrow \infty} \omega(t) \leq \omega\left(\alpha_{n}\right)-\limsup _{t \rightarrow \infty}\left[\int_{\alpha_{n}}^{t}\left(R(s) d s+\sum_{\alpha_{n} \leq \theta_{k}<t} \rho^{\alpha}\left(\theta_{k}\right) q\left(\theta_{k}\right)\right)\right]<0 .
$$

This implies that $u^{\prime}(t)<0$ for all $t \geq \alpha_{n}$ which is a contradiction.

Case 3: $u^{\prime}(t)<0$ for $t \geq t_{1}$. Because of condition (3) we obtain a contradiction from the proof of Theorem 1. This completes the proof.

Theorem 4. Assume that conditions $\left(H_{1}\right),\left(H_{2}\right),\left(H_{4}\right)$ and $(2)$ hold with $\psi(u) \equiv 1$. If $\rho(t)$ is a positive non-decreasing differentiable function such that

$$
\lim _{t \rightarrow \infty} \int_{t_{0}}^{t}\left(\frac{e^{-\int_{t_{0}}^{s} \frac{p(u)}{r(u)} d u}}{\rho^{\alpha}(s) r(s)}\right)^{\frac{1}{\alpha}} d s=\infty
$$

and

$$
\limsup _{t \rightarrow \infty} \int_{t_{0}}^{t} R^{*}(s) d s+\sum_{t_{0} \leq \theta_{k}<t} \rho^{\alpha}\left(\theta_{k}\right) q\left(\theta_{k}\right)=\infty
$$

where

$$
R^{*}(s)=\rho^{\alpha}(t)\left[q(t)-\mu_{1} r(t)\left|\frac{\alpha \rho^{\prime}(t)}{\rho(t)}+\frac{p(t)}{r(t)}\right|^{\alpha+1}\right]
$$

and $\mu_{1}:=\frac{1}{(\alpha+1)^{\alpha+1}}\left(\frac{\alpha}{L}\right)^{\alpha}$, then every solution of equation (1) is oscillatory.

Proof. Let $u(t)$ be a non-oscillatory solution of equation (1). Without loss of generality we may assume that $u(t)>0$ for all $t \geq t_{1} \geq t_{0}$. Differentiating (5) 
and using $\left(H_{2}\right),\left(H_{4}\right)$ and $(2)$, and then proceeding as in the proof of Theorem 1 , we obtain

$$
\omega^{\prime}(t) \leq \rho^{\alpha}(t)\left[q(t)-\mu_{1} r(t)\left|\frac{\alpha \rho^{\prime}(t)}{\rho(t)}+\frac{p(t)}{r(t)}\right|^{\alpha+1}\right] .
$$

Integrating the inequality (21) from $t_{1}$ to $t$, and using (11), we have

$$
\begin{gathered}
\omega(t) \leq \omega\left(t_{1}\right)-\left[\int_{t_{1}}^{t} \rho^{\alpha}(s)\right. \\
\left(q(s)-\mu_{1} r(s)\left|\frac{\alpha \rho^{\prime}(s)}{\rho(s)}+\frac{p(s)}{r(s)}\right|^{\alpha+1}\right) d s \\
\left.+\sum_{t_{1} \leq \theta_{k}<t} Q_{k} \frac{\rho^{\alpha}\left(\theta_{k}\right)}{f\left(u\left(\theta_{k}\right)\right)}\right]
\end{gathered}
$$

or

$$
\omega(t) \leq \omega\left(t_{1}\right)-\left[\int_{t_{1}}^{t} R^{*}(s) d s+\sum_{t_{1} \leq \theta_{k}<t} q\left(\theta_{k}\right) \rho^{\alpha}\left(\theta_{k}\right)\right] .
$$

Taking $\limsup _{t \rightarrow \infty}$ in the last inequality, we obtain $\omega(t) \rightarrow-\infty$ in view of (20). Hence there exists $t_{2} \geq t_{1}$ such that $u^{\prime}(t)<0$ for $t \geq t_{2}$. Then Proceeding similarly as in Theorem 1 and using condition (19), we obtain a contradiction. The proof is complete.

The following result is an immediate consequence of Theorem 4.

Corollary 5. Assume all the conditions of Theorem 4 are satisfied except (20). If

$$
\limsup _{t \rightarrow \infty} \int_{t_{0}}^{t} \rho^{\alpha}(s) q(s) d s+\sum_{t_{0} \leq \theta_{k}<t} \rho^{\alpha}\left(\theta_{k}\right) q\left(\theta_{k}\right)=\infty,
$$

and

$$
\limsup _{t \rightarrow \infty} \int_{t_{0}}^{t} \rho^{\alpha}(s) r(s)\left|\frac{\alpha \rho^{\prime}(s)}{\rho(s)}+\frac{p(s)}{r(s)}\right|^{\alpha+1} d s<\infty
$$

then every solution of equation (1) with $\psi(u)=1$ is oscillatory.

We conclude this section with the following Theorem.

Theorem 6. Assume that $\left(H_{1}\right),\left(H_{2}\right),\left(H_{4}\right)$ and $(2),(18)$ hold and $\psi(u) \equiv$ 1 . If there exists a differentiable function $\rho:\left[t_{0}, \infty\right) \rightarrow \mathbb{R}^{+}$with (19) holds. If

$$
\int_{t_{0}}^{\infty} \rho^{\alpha}(s) q(s) d s+\sum_{t_{0} \leq \theta_{k}<t} \rho^{\alpha}\left(\theta_{k}\right) q\left(\theta_{k}\right)<\infty,
$$


and

$$
\liminf _{t \rightarrow \infty}\left[\int_{t_{0}}^{t} R^{*}(s) d s+\sum_{t_{0} \leq \theta_{k}<t} \rho^{\alpha}\left(\theta_{k}\right) q\left(\theta_{k}\right)\right] \geq 0
$$

and

$$
\lim _{t \rightarrow \infty} \int_{t_{0}}^{t}\left(\frac{1}{r(s) \rho^{\alpha}(s)}\left[\int_{s}^{\infty} R^{*}(u) d u+\sum_{s \leq \theta_{k}<\infty} \rho^{\alpha}\left(\theta_{k}\right) q\left(\theta_{k}\right)\right]\right)^{\frac{1}{\alpha}} d s=\infty
$$

then every solution of equation (1) is oscillatory.

Proof. Proof is similar to that of Theorem 3, and hence the details are omitted.

\section{Examples}

Example 6.1. Consider the following second order impulsive differential equation

$$
\left\{\begin{array}{l}
\left(\frac{1}{t^{2}} \frac{1}{u^{4}}\left|u^{\prime}\right| u^{\prime}\right)^{\prime}+\left(\frac{3}{2} t^{\frac{-3}{2}}\left(2+t^{2}\right)+t e^{u}\right) u=u t^{\frac{-7}{2}}+t^{-5} \frac{u}{u^{2}+1}, t \geq 1, t \neq 2^{k} \\
\left.\Delta\left(\frac{1}{t^{2}} \frac{1}{u^{3}}\left|u^{\prime}\right| u^{\prime}\right)\right|_{t=2^{k}}+\left(\frac{3}{2}\left(2^{k}\right)^{\frac{-3}{2}}\left(2+2^{2 k}\right)+2^{k} e^{u}\right) u=0, t=2^{k}, k=1,2 \ldots
\end{array}\right.
$$

Here $r(t)=\frac{1}{t^{2}}, \theta_{k}=2^{k}, t_{0}=1, \psi(u)=\frac{1}{u^{4}}, \alpha=2$. If we choose $f(x)=x$ and $\rho(t)=t$, then

$$
\begin{aligned}
\frac{Q(t, u)}{f(u)} & \geq \frac{3}{2} t^{-\frac{3}{2}}\left(2+t^{2}\right)+t e^{u} \geq \frac{3}{2} t^{-\frac{3}{2}}\left(2+t^{2}\right)=q(t), \\
\frac{P\left(t, u, u^{\prime}\right)}{f(u)} & \leq t^{-\frac{7}{2}}+\frac{1}{t^{5}} \frac{1}{u^{2}+1} \leq t^{-\frac{7}{2}}+\frac{1}{t^{5}}=p(t) .
\end{aligned}
$$

Now, it is easy to see that all conditions of Theorem 1 are satisfied and hence every solution of equation (28) is oscillatory.

Example 6.2. Consider the following second order impulsive differential equation 


$$
\left\{\begin{array}{l}
\left(\frac{1}{t} u^{-2} u^{\prime}\right)^{\prime}+\frac{1}{t^{3}} u^{3}=\frac{1}{4 t^{3}} u^{3}, t \geq 1, t \neq 2^{k} \\
\left.\Delta\left(t^{-1} u^{-2} u^{\prime}\right)\right|_{t=2^{k}}+\left(2^{k}\right)^{-3} u^{3}\left(2^{k}\right)=0, t=2^{k}, k=1,2 \ldots
\end{array}\right.
$$

Here $r(t)=\frac{1}{t}, \theta_{k}=2^{k}, t_{0}=1, \psi(u)=u^{-2}, \alpha=1$. If we choose $f(x)=x^{3}$ and $\rho(t)=t$, then

$$
\frac{Q(t, u)}{f(u)} \geq \frac{1}{t^{3}}=q(t), \frac{P\left(t, u, u^{\prime}\right)}{f(u)} \leq \frac{1}{4 t^{3}}=p(t) .
$$

Now, it is easy to see that all conditions of Theorem 3 are satisfied and hence every solution of equation (28) is oscillatory.

Remark 6.1. The results obtained in this paper reduces to that of [7] when the impulse are dropped.

Remark 6.2. It would be interesting to extend the results obtained in this paper to delay and neutral type impulsive differential equations.

\section{Acknowledgments}

Second author gratefully acknowledges the Research Fellowship granted by the University Grants Commission of India, for Meritorious students in Sciences.

\section{References}

[1] J. R. Graef, S. M. Rankin and P. W. Spikes, Oscillation theorems for perturbed non-linear differential equation,J. Math. Anal. Appl.,65 (1978), 375-390.

[2] V. Lakshmikantham, D. Bainov and P.S. Simenov, Theory of Impulsive Differential Equaions, World Scientific Pub. Co., Inc., NJ, 1989.

[3] X. Li , Oscillation properties of second order delay differential-difference equations with impulses, Adv. Appl. Math. Anal.,3 (2008), 55-66.

[4] W. Li, C. Yang and H. Ji, Oscillation of a class of second order nonlinear ordinary differential equation with impulses, Appl. Math. Comput., 138 (2003), 181-188. 
[5] M. Peng and W. Ge, Oscillation criteria for second order nonlinear differential equation with impulses, Comput. Math. Appl., 39 (2000), 217-255.

[6] M. Remili, Oscillation criteria for second order nonlinear perturbed differential equation,Electron. J. Qual. Theory. Diff. Eqn., 25 (2010), 1-11.

[7] P. Temtex and A. Tiryaki, Oscillation criteria for a certain second- order nonlinear perturbed differential equations, J. Inequal. Appl, 2013, 2013:524.

[8] E.Thandapani, R.Sakthivel and E.Chandrasekaran, Oscillation criteria for second order nonlinear perturbed impulsive diferential equations, J. Appl. Math. Inform., .

[9] J. Yan, Oscillation properties of a second order impulsive delay differential equation, Com. Math. Appl., 47 (2004), 253-258.

[10] Q. Zhang and L. Wang, Oscillatory behavior of solutions for a class of second order nonlinear differential equation with perturbation, Acta. Appl. Math., 110 (2010), 885-893.

[11] Q. Zhang, F. Qiu and L. Gao, Oscillatory property of solutions for a class of second order nonlinear differential equation with perturbation, J. Appl. Math. Inform., 28 (2010), 883-892.

[12] Y. Zhang and A.Zhao, Oscillation criteria for impulsive delay differential equations,J. Math. Anal. Appl., 205 (1997), 461-470. 